\title{
The boson-fermion model with on-site Coulomb repulsion between fermions
}

\author{
Alfonso Romano \\ Dipartimento di Fisica "E.R. Caianiello", Università di Salerno, I-84081 Baronissi (Salerno), Italy \\ Unità I.N.F.M. di Salerno
}

(November 1, 2018)

\begin{abstract}
The boson-fermion model, describing a mixture of itinerant electrons hybridizing with tightly bound electron pairs represented as hard-core bosons, is here generalized with the inclusion of a term describing on-site Coulomb repulsion between fermions with opposite spins. Within the general framework of the Dynamical Mean-Field Theory, it is shown that around the symmetric limit of the model this interaction strongly competes with the local boson-fermion exchange mechanism, smoothly driving the system from a pseudogap phase with poor conducting properties to a metallic regime characterized by a substantial reduction of the fermionic density. On the other hand, if one starts from correlated fermions described in terms of the one-band Hubbard model, the introduction in the half-filled insulating phase of a coupling with hard-core bosons leads to the disappearance of the correlation gap, with a consequent smooth crossover to a metallic state.
\end{abstract}

PACS numbers: 71.10.Fd, 71.30.+h, 71.10.Fd, 74.20.Mn

A large variety of phenomena in condensed matter physics has been studied in the past in terms of interacting models with coupled bosonic and fermionic degrees of freedom [1]. Their relevance has proved to be of particular evidence in the analysis of the electron-phonon problem and of the related issue of the polaron and bipolaron formation. In this context, it was proposed some years ago [2] that for a system of itinerant electrons interacting with local lattice deformations, the crossover regime between adiabatic and non-adiabatic behavior can be described by a model where tightly bound electron pairs (hard-core bosons) of polaronic origin coexist with quasifree electrons (fermions), with an exchange coupling assumed between them by which bosons can decay into pairs of itinerant fermions and vice-versa. It has subsequently been suggested [3] that this Boson-Fermion Model (BFM) can provide a possible scenario for high$T_{c}$ superconductivity, according to the hypothesis that the fermionic degrees of freedom describe holes confined in the copper-oxygen planes, while the bosonic ones are associated with bipolarons which form in the highly polarizable dielectric layers sandwiching the $\mathrm{CuO}_{2}$ planes. Since then, the model has been deeply investigated, in particular by Ranninger and coworkers [4 [6], with a special attention to the description of the pseudogap phase characterizing the normal state of the underdoped high$T_{c}$ copper oxides.

In this paper we consider an extension of the BFM in which fermions, besides exchanging with bosons, are assumed to also experience an on-site Coulomb repulsion. This interaction, tending to prevent two fermions with opposite spin from occupying the same site, strongly competes with the boson-fermion exchange mechanism, giving rise to interesting effects in the spectral as well as in the transport properties. The model, whose fermionic part goes back in the one-band Hubbard model in the limit of vanishing boson-fermion coupling, is solved here within the framework of the Dynamical Mean-Field Theory (DMFT) [7 11, by applying to the related selfconsistent impurity problem the so-called Non-Crossing Approximation (NCA) [12]. We recall that DMFT-based approaches have been intensively used in the last years in the related context of electron-phonon systems described by the Holstein model [13,14] or extensions of it [15, as well as in the case of boson-fermion coupled systems with boson dispersion 16. By means of various analytical and numerical techniques, a special attention has also been devoted to the role played by the on-site Coulomb repulsion in the electron-phonon problem and, in particular, on the polaron and bipolaron formation. This has been done essentially referring to a generalized version of the Holstein model which includes an Hubbard interaction term (the so called Holstein-Hubbard model) 17.

The hamiltonian for the BFM with Coulomb repulsion between fermions is given by

$$
\begin{aligned}
H= & \varepsilon_{0} \sum_{i, \sigma} c_{i \sigma}^{\dagger} c_{i \sigma}-t \sum_{\langle i j\rangle, \sigma} c_{i \sigma}^{\dagger} c_{j \sigma}+U \sum_{i} c_{i \uparrow}^{\dagger} c_{i \uparrow} c_{i \downarrow}^{\dagger} c_{i \downarrow} \\
& +E_{0} \sum_{i} b_{i}^{\dagger} b_{i}+g \sum_{i}\left[b_{i}^{\dagger} c_{i \downarrow} c_{i \uparrow}+c_{i \uparrow}^{\dagger} c_{i \downarrow}^{\dagger} b_{i}\right] .
\end{aligned}
$$

Here $c_{i \sigma}^{(\dagger)}$ denote annihilation (creation) operators for electrons with spin $\sigma$ at site $i$ and $b_{i}^{(\dagger)}$ denote hard-core bosonic operators describing tightly bound localized electron pairs. The site energies for fermions and bosons are expressed as $\varepsilon_{0}=D-\mu$ and $E_{0}=\Delta_{B}-2 \mu$, respectively, where the chemical potential $\mu$ is assumed to be common to the two kinds of carriers (up to a factor 2 for the bosons) in order to guarantee charge conservation. Finally, $g$ denotes the boson-fermion pair-exchange coupling constant, while $t, D$, and $U$ are respectively the bare hopping integral, the bare half bandwidth and the on-site Coulomb repulsion, all referred to fermions. We see that in the limit of vanishing Coulomb repul- 
sion the model goes back in the boson-fermion model for high- $T_{c}$ superconductors analyzed in Refs. [3 6 . On the other hand, in the limit of zero boson-fermion coupling the fermionic part of the model defined by the Hamiltonian (1) coincides with the standard one-band Hubbard model.

It is worth pointing out that the assumption of an on-site Coulomb interaction affecting only the fermionic degrees of freedom is motivated by the existence of anisotropic systems, such as the previously mentioned high- $T_{c}$ copper oxides, where there is experimental evidence that bosons and fermions reside in separated lattice regions where Coulomb effects are in general significantly different. In this respect, the site indices in the phenomenological Hamiltonian (1) would better be thought as representative of molecular units composed, for instance, by an atom on which the correlated fermions sit and two adjacent ones forming a diatomic molecule housing the bosonic tightly bound electron pairs. Given this picture, no direct interference occurs between the on-site fermionic Coulomb repulsion and the bosonic formation, the only effect being a shift at higher energies of the fermionic level with respect to the bosonic one, leading to a relative change in occupation of the two species. The assumption of a lattice seen as a collection of effective sites is of course made to simplify the analytical as well as the numerical calculations, and a more accurate (though technically more complicate) way of analyzing the role of the Coulomb repulsion would require the solution of the model on a true bipartite lattice.

Given the local character of the interaction terms in the Hamiltonian (1), important insights on the physics of the model can be obtained from its atomic limit. The eigenstates and eigenvalues of $H$ are in this case given by

$$
\begin{array}{ll}
|1\rangle=|0\rangle & E_{1}=0 \\
|2\rangle=|\uparrow\rangle & E_{2}=\varepsilon_{0} \\
|3\rangle=|\downarrow\rangle & E_{3}=\varepsilon_{0} \\
|4\rangle=u|\uparrow \downarrow\rangle-v|\bullet\rangle & E_{4}=\varepsilon_{0}+U / 2+E_{0} / 2-\gamma \\
|5\rangle=v|\uparrow \downarrow\rangle+u|\bullet\rangle & E_{5}=\varepsilon_{0}+U / 2+E_{0} / 2+\gamma \\
|6\rangle=|\uparrow \bullet\rangle & E_{6}=\varepsilon_{0}+E_{0} \\
|7\rangle=|\downarrow \bullet\rangle & E_{7}=\varepsilon_{0}+E_{0} \\
|8\rangle=|\uparrow \downarrow \bullet\rangle & E_{8}=2 \varepsilon_{0}+U+E_{0}
\end{array}
$$

where the dot indicates the presence of a boson and we have defined

$$
\begin{aligned}
u^{2} & =\frac{1}{2}\left[1-\frac{2 \varepsilon_{0}+U-E_{0}}{2 \gamma}\right] \\
\gamma & =\frac{1}{2}\left[\left(2 \varepsilon_{0}+U-E_{0}\right)^{2}+4 g^{2}\right]^{1 / 2}
\end{aligned}
$$

with $u^{2}+v^{2}=1$. When $U=0$ and the total particle density $n=n_{F}+2 n_{B}$ is equal (or close) to 2 , the physics underlying the model is fully determined by the charge exchange interaction. As the temperature is decreased, the spectral weight of the pole in the Green's function associated with the non-bonding single-particle state (at energy $\varepsilon_{0}$ ) is gradually transferred to the poles (at energies $E_{4}-\varepsilon_{0}$ and $\left.E_{5}-\varepsilon_{0}\right)$ associated with the bonding and anti-bonding two-particle states $\mid 4>$ and $\mid 5>[18$. The corresponding effect seen when itinerancy is taken into account, is a depletion of the density of states in the region around the chemical potential. This gives rise to the opening a pseudogap which, according to the mechanism explained above, is thus induced by local pairing among the electrons.

When the effect of the on-site Coulomb repulsion among fermions is introduced, the structure of the atomic Green's function becomes more complex, with the number of poles increasing from 3 to 6 . The transfer of spectral weight from single-particle to two-particle resonances as $T$ is decreased, becomes less and less pronounced as higher values of $U$ are considered. As expected, this is a consequence of the fact that a finite $U$ tends to forbid double fermion occupations of the same site, thus inhibiting the boson-fermion charge exchange mechanism. More precisely, what one can see from the atomic limit solution is that, upon increasing $U, u^{2}$ tends to zero, $v^{2}$ tends to 1 , and thus the two states $\mid 4>$ and $\mid 5>$ tend to become pure one-boson and two-fermion states, respectively, with a separation in energy of the order of $U$. As we will see in the following, for the full model around the symmetric limit this has the simultaneous effect of hindering the opening of the pseudogap and making the bosonic site occupation considerably higher than the fermionic one.

The effect of the electron itinerancy will now be taken into account within the general framework of the Dynamical Mean-Field Theory (DMFT) [7 11]. In the last years this approach has proved to be of fundamental importance in condensed matter physics and has been successfully applied to a variety of many-body models. Within DMFT an interacting system is seen as a purely local system coupled to a Weiss "mean field" to be determined self-consistently, produced by the neighboring sites. This approach leads to the freezing of all spatial fluctuations, just like the usual classical mean-field theory, but has the advantage of fully retaining the quantum temporal fluctuations of the original problem (hence the adjective "dynamical"). Indeed, the mean field is here a function of time, instead of being a pure number, associated with the probability amplitude of creating a particle on the impurity center at a given time and destroying back in the external bath at a later time, and vice-versa. Like any mean field approximation, this approach becomes more and more accurate as the lattice coordination number increases, becoming exact in the limit of infinite dimensionality. This basic structure makes the application of the DMFT particularly suited to models, such as that defined by the Hamiltonian (1), where the interaction terms are local. A method for the inclusion of non-local corrections 
to DMFT, known as Dynamical Cluster Approximation, has recently been developed [19] and applied to the twodimensional Hubbard model [20], by mapping the lattice problem onto a periodic cluster embedded in an external host.

Since we consider here a model where correlated electrons are coupled to dispersionless bosons, in the DMFT self-consistent procedure the bosonic channel for the Green's function remains frozen, in the sense that the related bare Weiss self-energy at each iteration is unrenormalized and fixed at its initial non-interacting expression. This point, discussed at length in Ref. [16], implies that the general dynamical mean-field framework in which the model defined by the Hamiltonian (1) is investigated, is essentially the same as that developed for the Hubbard model in Refs. [7 9], the effect of the bosonfermion exchange coupling manifesting itself only in the solution of the impurity problem. In the following we give a brief summary of the DMFT technique, along the general lines of the diagramatic approach presented in Refs. [8,9]. The reader is referred to these papers, as well as to Refs. [7, 10, 11], for all technical details not reported here for brevity.

The approach starts from the demonstration 21.22 that in the limit of infinite dimensionality only the local dynamics remains nontrivial, in the sense that the only non-vanishing contributions to the self-energy are the site-diagonal ones. As a consequence, the Dyson equation in real space takes the form

$$
G_{i j}\left(i \omega_{n}\right)=G_{i j}^{0}\left(i \omega_{n}\right)+\sum_{l} G_{i l}\left(i \omega_{n}\right) \Sigma\left(i \omega_{n}\right) G_{l j}\left(i \omega_{n}\right)
$$

When this equation is referred to the local propagator $G_{i i}$, one can first consider the self-energy contributions coming from all sites $j$ other than the site $i$. The resummation of these contributions lead to the definition of a modified propagator, that we denote by $G_{W}$ (the Weiss field), that is then used in the full Dyson equation for $G_{i i}$, reintroducing the missing self-energy contributions that involve the same site $i[9]$. This procedure leads to the following Dyson equation for $G_{i i} \equiv G_{i m p}$

$$
G_{i m p}\left(i \omega_{n}\right)=\left[G_{W}^{-1}\left(i \omega_{n}\right)-\Sigma\left(i \omega_{n}\right)\right],
$$

which proves the equivalence of the lattice problem to a model of an Anderson impurity self-consistently embedded in a medium specified by the Weiss Green's function $G_{W}$. Given for the latter the structure $G_{W}\left(i \omega_{n}\right)=$ $\left[i \omega_{n}-\varepsilon_{0}-\Sigma_{W}\left(i \omega_{n}\right)\right]^{-1}$, the above equation implies that the impurity Green's function can be expressed in the form

$$
G_{i m p}\left(i \omega_{n}\right)=\frac{1}{i \omega_{n}-\varepsilon_{0}-\Sigma_{W}\left(i \omega_{n}\right)-\Sigma_{i n t}\left(i \omega_{n}\right)},
$$

where we have put $\Sigma_{i n t} \equiv \Sigma$. We thus see that the total self-energy is written as the sum of two momentumindependent contributions $\Sigma_{i n t}$ and $\Sigma_{W}$, associated respectively with the on-site interactions (the bosonfermion exchange and the Coulomb repulsion among fermions) and with the hybridization of the impurity center with the medium.

On the other hand, due to the locality of the selfenergy, the lattice Green's function in $\mathbf{k}$-space is given by

$$
G_{l a t}\left(\varepsilon_{\mathbf{k}}, i \omega_{n}\right)=\frac{1}{i \omega_{n}-\varepsilon_{0}-\varepsilon_{\mathbf{k}}-\Sigma_{i n t}\left(i \omega_{n}\right)}
$$

where $\varepsilon_{\mathbf{k}}$ denotes the bare electron dispersion. The self-consistency condition which iteratively leads to the renormalization of the Weiss self-energy, thus closing the DMFT algorythm, is then simply obtained by equating the impurity Green's function (6) to the integral over kspace of the lattice Green's function, i.e. $G_{i m p}\left(i \omega_{n}\right)=$ $N^{-1} \sum_{\mathbf{k}} G_{l a t}\left(\varepsilon_{\mathbf{k}}, i \omega_{n}\right)$. Upon replacement of the integration over $\mathbf{k}$ by an integration over energy, this condition takes the form

$$
G_{i m p}\left(i \omega_{n}\right)=\int d \varepsilon \frac{\rho(\varepsilon)}{i \omega_{n}-\varepsilon_{0}-\varepsilon-\Sigma_{i n t}\left(i \omega_{n}\right)},
$$

where $\rho$ is the free density of states associated with the particular lattice chosen.

The whole DMFT procedure can thus be summarized as follows. One starts with a guess for the Weiss Green's function $G_{W}$ and solve the corresponding impurity problem, obtaining $G_{i m p}$ (here this is done by means of the socalled Non-Crossing Approximation). The resulting local self-energy $\Sigma_{i n t} \equiv \Sigma$, evaluated from Eq.(5), is then used to find a new impurity Green's function by imposing the self-consistency condition (8). By subtracting off from the latter the local self-energy, again by use of Eq.(5), one gets a new Weiss field which is used to reinitialize the process, which is then iterated until convergence is reached.

Let us also notice that the explicit form taken by the self-consistency condition depends on the particular lattice on which the DMFT equations are solved. In the following we assume to refer to a Bethe lattice with coordination number $z \rightarrow \infty$ and nearest neighbor hopping $t_{i j}=t / \sqrt{z}$, for which the electronic bare density of states is the semi-circular function $\rho(\varepsilon)=\left(1 / 2 \pi t^{2}\right) \sqrt{4 t^{2}-\varepsilon^{2}}$ $(|\varepsilon|<2 t)$ of width $2 D=4 t$. This assumption, widely used in the context of the DMFT [14 [16,23,24] allows to solve analytically eq.(8), leading to

$$
\Sigma_{W}\left(i \omega_{n}\right)=t^{2} G_{i m p}\left(i \omega_{n}\right)
$$

or, in terms of the corresponding imaginary parts,

$$
\Delta(\omega)=t^{2} A_{F}(\omega)
$$


(we use the definition $\Delta(\omega)=-2 \operatorname{Im} \Sigma_{W}(\omega+i \eta)$, with a similar equation relating $A_{F}$ and $\left.G_{i m p}\right)$.

The single-site effective dynamics described above is naturally investigated by means of functional methods based on the use of an imaginary-time effective action for the fermionic degrees of freedom defined at the impurity center [7,10]. An equivalent picture can be introduced by considering a related Hamiltonian formulation where the Weiss field is described in terms of auxiliary degrees of freedom. In the case of the BFM, we have an effective Hamiltonian of the form

$$
\begin{aligned}
\widetilde{H}= & \sum_{\sigma} \varepsilon_{0} c_{\sigma}^{\dagger} c_{\sigma}+U c_{\uparrow}^{\dagger} c_{\uparrow} c_{\downarrow}^{\dagger} c_{\downarrow} \\
& +E_{0} b^{\dagger} b+g\left[c_{\uparrow}^{\dagger} c_{\downarrow}^{\dagger} b+b^{\dagger} c_{\downarrow} c_{\uparrow}\right] \\
& +\sum_{k, \sigma} w_{k} d_{k \sigma}^{\dagger} d_{k \sigma}+\sum_{k, \sigma} v_{k}\left[d_{k \sigma}^{\dagger} c_{\sigma}+c_{\sigma}^{\dagger} d_{k \sigma}\right]
\end{aligned}
$$

where $c_{\sigma}^{(\dagger)}$ and $b^{(\dagger)}$ denote the original fermion and boson operators, respectively, at the selected site and $d_{k \sigma}^{(\dagger)}$ are the auxiliary fermionic operators associated with the Weiss field, taking into account the dynamics occurring at all the other sites. Their energy spectrum $w_{k}$ and their coupling $v_{k}$ to the impurity electrons are of course not known a priori but must be determined self-consistently. Identifying the Weiss Green's function with the propagator for the auxiliary variables, one gets

$$
G_{W}^{-1}\left(i \omega_{n}\right)=i \omega_{n}-\varepsilon_{0}-\sum_{k} \frac{v_{k}^{2}}{i \omega_{n}-w_{k}}
$$

with the sum at the right-hand side representing the selfenergy $\Sigma_{W}$. We thus see that the Hamiltonian description is fully equivalent to the functional one provided that the parameters $v_{k}$ and $w_{k}$ are related by the following equation to the imaginary part $\Delta(\omega)$ of the Weiss self-energy appearing in the effective action:

$$
\Delta(\omega)=2 \pi \sum_{k} v_{k}^{2} \delta\left(\omega-w_{k}\right)
$$

Depending on the technique adopted to solve the impurity problem, the Hamiltonian formulation can prove to be useful in practical calculations, besided providing a description probably closer to our physical intuition.

The self-consistent single-impurity Anderson problem defined by the effective Hamiltonian (11) is solved here within the so-called Non Crossing Approximation (NCA) [12], along the lines presented and discussed in Ref. [5]. Within the DMFT framework, NCA has been applied in the last years to several other models, such as the oneband Hubbard model [25], a multiband Hubbard model for perovskites [26], the Anderson lattice model with correlated conduction electrons [23], and the Kondo lattice model with correlated conduction electrons 24]. In a very recent paper this technique has also been employed to analyze the electronic structure of the ruthenate alloy series $\mathrm{Ca}_{2-x} \mathrm{Sr}_{x} \mathrm{RuO}_{4}$ 27.

All the results presented in the following refer to the case of a total particle density $n=n_{F}+2 n_{B}$ equal to 2 , with $n_{F}$ and $n_{B}$ being respectively computed from the fermionic and the bosonic impurity propagators. All energies are measured in units of the bare bandwidth $2 D$. In order to emphasize the effects of the competition between the boson-fermion coupling and the on-site Coulomb repulsion, we will mainly consider parameter regimes where $g$ and $U$ are comparable. We want also to point out that in these regimes the application of the conventional NCA approach is expected to give reliable results. This has been checked resorting to a validity criterion which is discussed at length in ref. [6] and which, for brevity, will not be repeated here. Finally, we have also verified that in the limit of vanishing boson-fermion coupling, the results for the Hubbard model obtained in Ref. [25], where the same DMFT+NCA algorythm is used, are correctly reproduced.

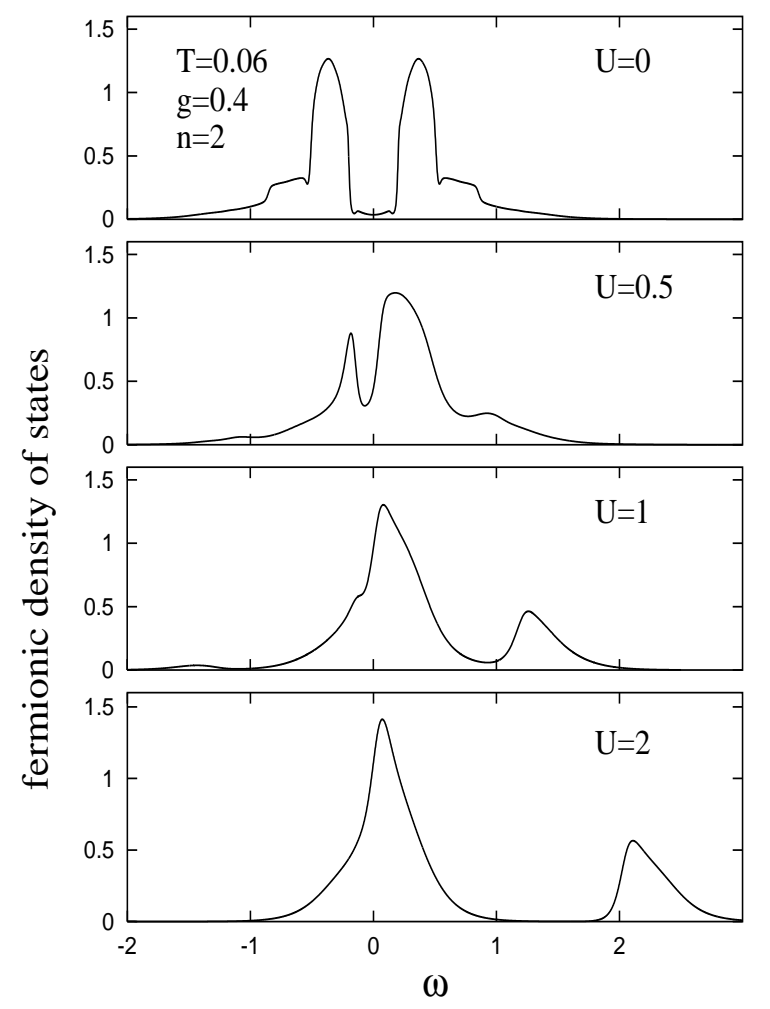

FIG. 1. Fermionic density of states for $T=0.06, g=0.4$ and several values of $U$. In the case $U=0$ the site energies satisfy the symmetric limit condition $E_{0}=\varepsilon_{0}=0$.

We start by discussing how the inclusion of $U$ affects the pseudogap phase as described by the BFM studied in Refs. [3 6]. In Fig.1 we have plotted the fermionic density of states for several values of the on-site repulsion (the energies are measured with respect to the chemical potential). We start at $U=0$ from the fully sym- 
metric case $E_{0}=\varepsilon_{0}=0$ (top panel), which implies $n_{F}=2 n_{B}=1$, and then consider finite values of $U$ while keeping the bosonic bare energy $\Delta_{B}$ fixed (the finite- $U$ symmetric limit conditions $E_{0}=2 \varepsilon_{0}+U=0$ are consequently not satisfied). We see that the widely open pseudogap showing up for $U=0$ is gradually filled as increasing values of $U$ are considered. At the same time an Hubbard correlation gap of the order of $U$ develops well beyond the chemical potential, thus without substantially affecting the low-energy physics. As already pointed out in the discussion of the atomic limit, this is due to the fact that the on-site repulsion, tending to inhibit the boson-fermion exchange mechanism, pushes the system in a regime where the coupling between the two components is reduced and, for the parameter choice considered here, the almost pure bosonic level remains pinned below the fermionic ones (these latter being splitted by $U$ ). Starting from the $U=0$ fully symmetric case considered in the upper panel, this also implies that much of the fermionic spectral weight is transferred at higher and higher energies as $U$ is increased, this leading to a substantial reduction of the fermionic occupation with respect to the bosonic one. We thus see that for the system is energetically more favourable to move away from the "insulating" half-filled case, creating holes in the fermionic configuration and thus allowing a smooth transition towards a metallic behavior.

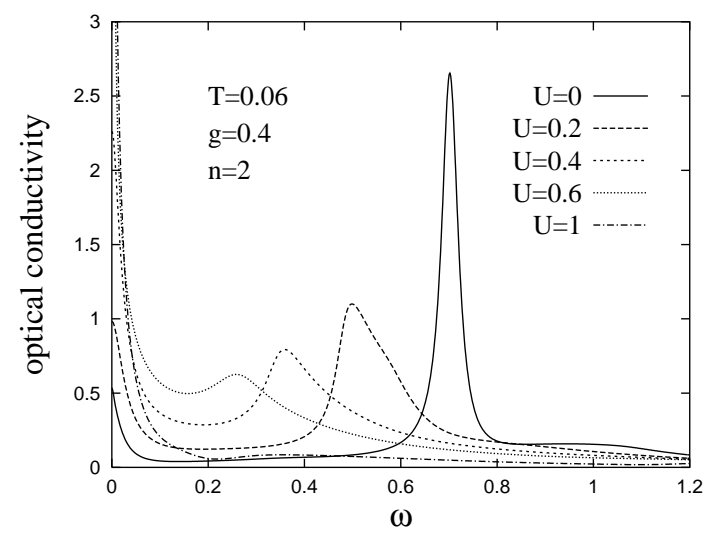

FIG. 2. Real part of the optical conductivity for the same parameters as in Fig.1 and several values of $U$.

These considerations find further support in the behavior of the real part of the optical conductivity, which in the limit of infinite dimensions becomes directly connected to the fermionic single-particle spectral function $A_{F}\left(\varepsilon_{k}, \omega\right)$ according to the relation

$$
\begin{aligned}
\sigma(\omega)= & \pi \int d \varepsilon \rho(\varepsilon) \int d \omega^{\prime} A_{F}\left(\varepsilon, \omega^{\prime}\right) A_{F}\left(\varepsilon, \omega+\omega^{\prime}\right) \times \\
& \times \frac{1}{\omega}\left[n_{F}\left(\omega^{\prime}\right)-n_{F}\left(\omega+\omega^{\prime}\right)\right]
\end{aligned}
$$

(here the sum over momenta has again been expressed as an energy integration over $\rho(\varepsilon)$ ). From the results re- ported in Fig.2 we see that a situation typical of that expected for an insulator is found for $U \rightarrow 0$, that is, we obtain a small d.c. conductivity $\sigma(0)$ with a peak at $\omega \simeq 2 g$ reflecting transitions between the bonding and the antibonding resonances exhibited by the density of states (see Fig.1). This peak gradually disappears as $U$ is increased, with a concomitant Drude-like accumulation of weight al low $\omega$, clearly associated with the establishment of metallic properties.

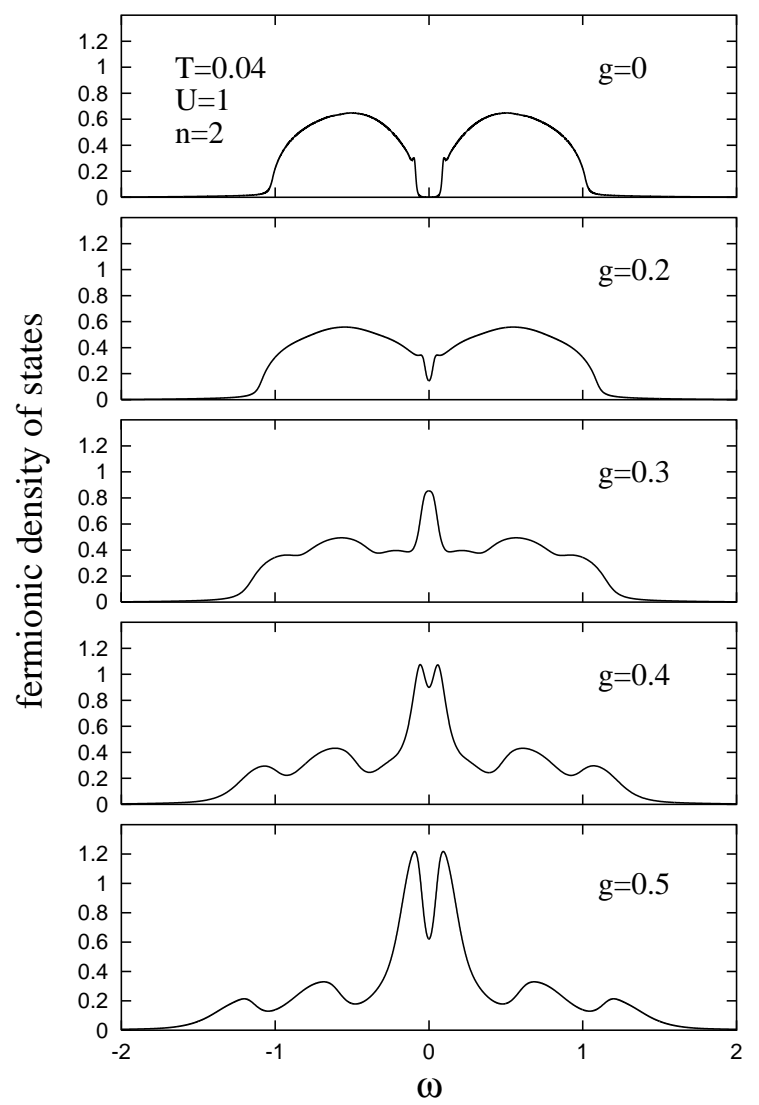

FIG. 3. Fermionic density of states in the symmetric limit $\left(E_{0}=2 \varepsilon_{0}+U=0\right)$ for $T=0.04, U=1$ and several values of $g$.

We have seen so far that if one starts from the symmetric limit of the BFM, characterized by a charge-exchange induced pseudogap in the excitation spectrum, then the introduction of the on-site Coulomb repulsion between fermions leads to a transition from an insulating state to a metallic state with lower carrier density. Recalling that in the limit $g=0$ the fermionic part of the model defined by the Hamiltonian (11) becomes equal to the Hubbard model, we now reverse the point of view, analyzing how the physics of the Hubbard model in the half-filled insulating phase is modified by the inclusion of the bosonfermion coupling. To this purpose, we assume to start from a parameter regime where, in the absence of $g$, the correlation gap is fully openin a half-filled configuration. Then we turn on the effect of $g$ under the assumption 
that the bosonic energy level is superimposed just at the middle of the unperturbed fermionic band, in such a way to ensure a particle density equally distributed among fermions and bosons $\left(n_{F}=2 n_{B}=1\right)$. As previously mentioned, this is realized when the symmetric limit conditions $E_{0}=0$ and $2 \varepsilon_{0}+U=0$ are simultaneously satisfied. The corresponding behavior of the fermionic density of states and of the real part of the optical conductivity is illustrated in Figs 3 and 1 , respectively.

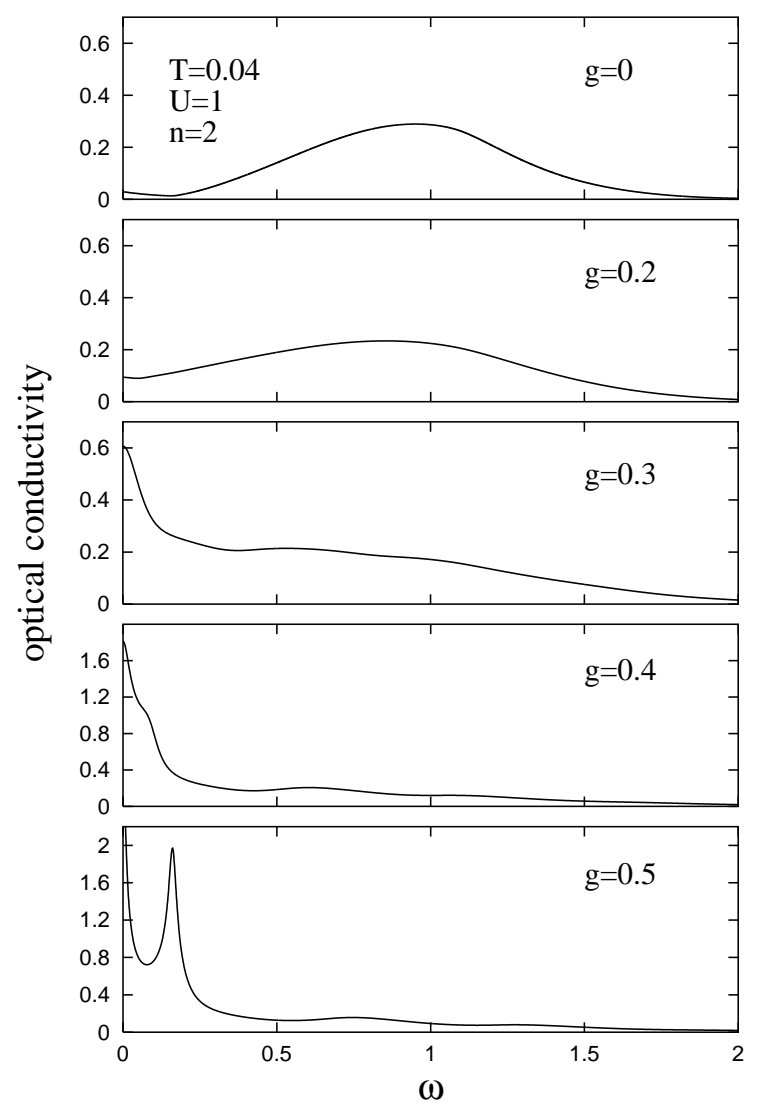

FIG. 4. Real part of the optical conductivity for the same parameters as in Fig. 3 .

We see that, as $g$ is increased from zero, the Hubbard gap is gradually closed as a consequence of the fact that the boson-fermion coupling tends to favor configurations with double fermionic occupations. As a result, this mechanism tends to create empty sites in the half-filled configuration, thus promoting the fermionic itinerancy. For suitably high values of $g$ a narrow pseudogap at the chemical potential tends to form again, driving back the system in a state where the metallic behavior is gradually weakened as $T$ is decreased. The substantial change in the transport properties induced by the boson-fermion coupling is also evident from the temperature behavior of the dc resistivity, plotted in Fig. difficulties our NCA algorythm is currently not able to provide stable enough results at temperatures lower than those considered in the figure).

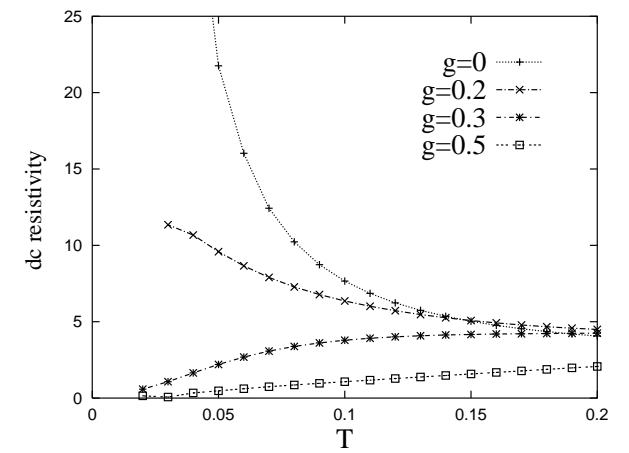

FIG. 5. Temperature dependence of the dc resistivity in the symmetric limit for $U=1$ and several values of $g$.

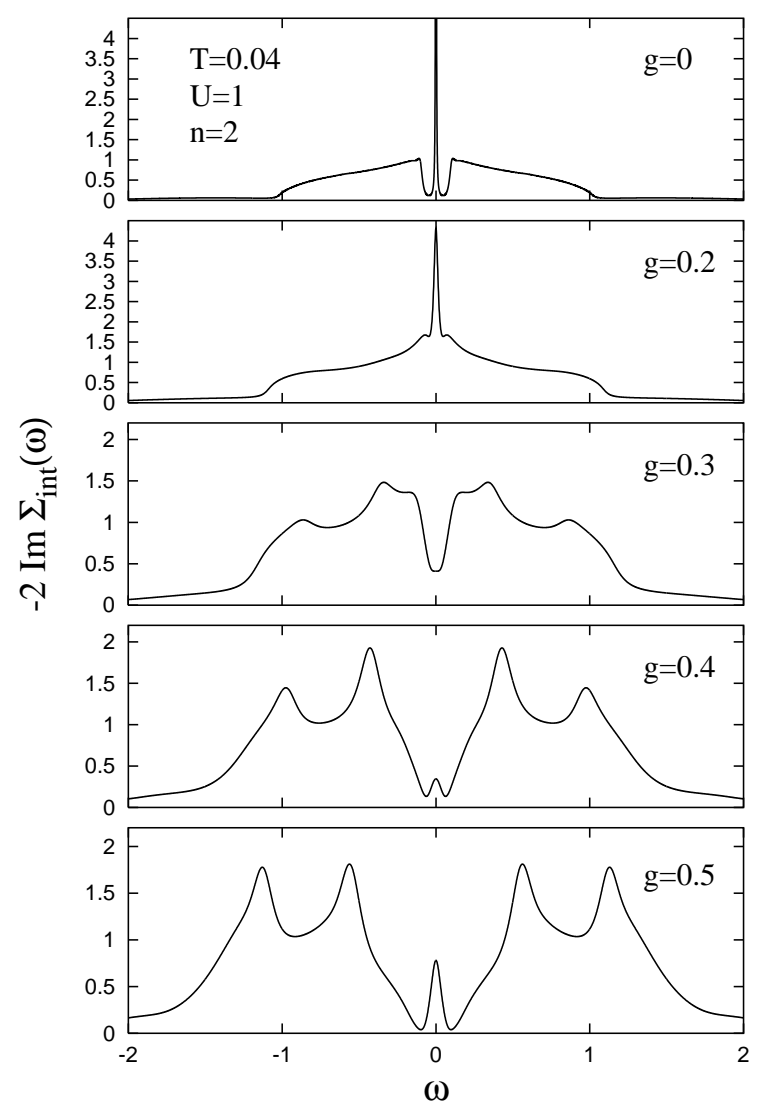

FIG. 6. Imaginary part of the fermionic self-energy for the same parameters as in Fig. 3 .

We can see that, while at $g=0$ and for low values of $g$ the system exhibits the expected semiconductor-like behavior, with $\sigma(0)^{-1}$ going up as $T$ is decreased, on the other hand higher values of $g$ give rise to a metalliclike behavior characterized by a decrease of $\sigma(0)^{-1}$ as $T \rightarrow 0$. Nonetheless, an important qualitative difference should be noted between the cases of intermediate and high values of $g$. The development of a dip at the chemical potential above approximately $g=0.4$ pushes the system toward a state which, though still showing metallic properties, is characterized by strong deviations from the Fermi liquid behavior. This is particularly evident 
when plotting the imaginary part of the fermionic selfenergy for the same parameter choices as in Fig.3. We can see from Fig. 6 that $-\operatorname{Im} \Sigma_{\text {int }}(\omega)$ exhibits for $g=0.3$ a parabolic minimum at $\mu$ (though with $\operatorname{Im} \Sigma(0)_{\text {int }} \neq 0$ ) which is the manifestation of a regime close to that of a normal Fermi liquid. When higher values of $g$ are considered, the behavior becomes significantly different, with the minimum being replaced by a narrow peak associated with a resonant scattering of the fermionic quasiparticles with the bosons.

In conclusion, we have analyzed a two-component model where correlated fermions and localized hard-core bosons interact through a mechanism by which bosons decay in two fermions with opposite spins and vice-versa. A special attention has been devoted to how the interplay between the boson-fermion coupling and the on-site Coulomb repulsion between fermions affects the spectral and the transport properties. Interesting crossovers from a pseudogap phase to a metallic regime, and vice-versa, have been found, as driven by the competition between the above mentioned local interactions. We emphasize that the way we have taken into account the effect of the on-site Coulomb interaction among fermions is not realistic if the model is applied to the description of high- $T_{c}$ superconductors. A more proper way would rather consist in defining the model on a lattice with two kinds of sites, one associated to the dielectric layers and the other one to the copper-oxygen planes. The Coulomb repulsion should then be introduced to describe correlation effects among holes in the latter, with the charge exchange mechanism now involving two strongly correlated fermions sitting on neighboring sites, rather than on the same site. A study along these lines is planned for the near future.

\section{Acknowledgements}

I would like to express my sincere thanks to J. Ranninger for all the stimulating discussions on the bosonfermion model that we had in the last years. I would also like to thank M. Cuoco and C. Noce for helpful discussions and a critical reading of the manuscript.

[1] See for instance: G.D. Mahan, Many Particle Physics (Plenum Pubishing, New York, 1981), and references therein.

[2] J. Ranninger and S. Robaszkiewicz, Physica B 135, 468 (1985).

[3] J. Ranninger, J.M. Robin, and H. Eschrig, Phys. Rev. Lett. 74, 4027 (1995).

[4] J. Ranninger and J.M. Robin, Phys. Rev. B 53, R11961 (1996); ibid. 56, 8330 (1997); P. Devillard and J. Ranninger, Phys. Rev. Lett. 84, 5200 (2000).
[5] J.M. Robin, A. Romano and J. Ranninger, Phys. Rev. Lett. 81, 2755 (1998).

[6] A. Romano and J. Ranninger, Phys. Rev. B 62, 4066 (2000).

[7] A. Georges and G. Kotliar, Phys. Rev. B 45, 6479 (1992).

[8] M. Jarrell, Phys. Rev. Lett. 69, 168 (1992).

[9] M. Jarrell and Th. Pruschke, Z. Phys. B 90, 187 (1993).

[10] A. Georges, G. Kotliar, W. Krauth and M. Rozenberg, Rev. Mod. Phys. 68, 13 (1996).

[11] F. Gebhard, "The Mott Metal-Insulator Transition" (Springer Verlag, Berlin, 1997).

[12] N.E. Bickers, Rev. Mod. Phys. 59, 845 (1987); N.E. Bickers, D.L. Cox and D.L. Wilkins, Phys. Rev. B 36, 2036 (1987); Th. Pruschke and N. Grewe, Z. Phys. B 74, 439 (1989).

[13] J.K. Freericks, M. Jarrell, and D.J. Scalapino, Phys. Rev. B 48, 6302 (1993) and Europhys. Lett. 25, 37 (1994); J.K. Freericks and M. Jarrell, Phys. Rev. B 50, 6939 (1994).

[14] S. Ciuchi, F. De Pasquale, S. Fratini, and D. Feinberg, Phys. Rev. B 56, 4494 (1997).

[15] A.J. Millis, R. Mueller, and B.I. Shraiman, Phys. Rev. B 54, 5389 (1996).

[16] Y. Motome and G. Kotliar, cond-mat/0005395.

[17] J. Takimoto and Y. Toyozawa, J. Phys. Soc. Japan 52, 4331 (1983); J. Zhong and H.B. Schüttler, Phys. Rev. Lett. 69, 1600 (1992); U. Trapper, H. Fehske, M. Deeg, and H. Büttner, Z. Phys. B 93, 465 (1994); G. Wellein, H. Röder, and H. Fehske, Phys. Rev. B 53, 9666 (1996); J.K. Freericks amd M. Jarrell, Phys. Rev. Lett. 75, 2570 (1995); T. Hotta and Y. Takada, Physica B 230-232, 1037 (1997); A. La Magna and R. Pucci, Phys. Rev. B 55, 14886 (1997); M. Capone, M. Grilli, and W. Stephan, cond-mat/9902317; J. Bonča, T. Katrašnik, and S.A. Trugman, Phys. Rev. Lett. 84, 3153 (2000); M. Acquarone, M. Cuoco, C. Noce, and A. Romano, Phys. Rev. B, 15 Jan. 2001 issue.

[18] T. Domanski, J. Ranninger and J.M. Robin, Solid State Commun. 105, 473 (1998).

[19] M.H. Hettler, A.N. Tahvildarzadeh, M. Jarrell, T. Pruschke, H.R. Krishnamurthy, Phys. Rev. B 58, 7475 (1998); M.H. Hettler, M. Mukherjee, M. Jarrell, and H.R. Krishnamurthy, Phys. Rev. B 61, 12739 (2000); Th. Maier, M. Jarrell, Th. Pruschke, and J. Keller, Eur. Phys. J. B 13, 613 (2000); Th. Maier, M. Jarrell, Th. Pruschke, and J. Keller, Phys. Rev. Lett. 85, 1524 (2000).

[20] M. Jarrell, Th. Maier, M.H. Hettler, and A.N. Tahvildarzadeh, cond-mat/0011282.

[21] W. Metzner amd D. Vollhardt, Phys. Rev. Lett. 62, 324 (1989).

[22] E. Müller-Hartmann, Z. Phys. B 74, 507 (1989).

[23] T. Schork and S. Blawid, Phys. Rev. B 56, 6559 (1997).

[24] T. Schork, S. Blawid, and J. Igarashi, Phys. Rev. B 59, 9888 (1999).

[25] Th. Pruschke, D.L. Cox and M. Jarrell, Phys. Rev. B 47, 3553 (1993).

[26] P. Lombardo, J. Schmalian, M. Avignon, and K.-H. Bennemann, Phys. Rev. B 54, 5317 (1997).

[27] V.I. Anisimov, I.A. Nekrasov, D.E. Kondakov, T.M. Rice, and M. Sigrist, cond-mat/0011460. 